\title{
Gradhiva
}

GRADHIV

Revue d'anthropologie et d'histoire des arts

$30 \mid 2019$

Précieux

\section{Anton Serdeczny, Du tabac pour le mort : une histoire de la réanimation, préface de Jean-Claude Schmitt} Ceyzérieu, Champ Vallon, coll. «Époques », 2018

\section{Vincent Guigueno}

\section{(2) OpenEdition}

\section{Journals}

Édition électronique

URL : http://journals.openedition.org/gradhiva/4777

DOI : $10.4000 /$ gradhiva. 4777

ISSN : 1760-849X

\section{Éditeur}

Musée du quai Branly Jacques Chirac

Édition imprimée

Date de publication : 4 décembre 2019

Pagination : 155

ISBN : 978-2-35744-113-2

ISSN : 0764-8928

\section{Référence électronique}

Vincent Guigueno, « Anton Serdeczny, Du tabac pour le mort: une histoire de la réanimation, préface de Jean-Claude Schmitt », Gradhiva [En ligne], 30 | 2019, mis en ligne le 31 mars 2021, consulté le 01 avril 2021. URL : http://journals.openedition.org/gradhiva/4777 ; DOI : https://doi.org/10.4000/gradhiva. 4777

Ce document a été généré automatiquement le 1 avril 2021.

(c) musée du quai Branly 


\section{Anton Serdeczny, Du tabac pour le mort : une histoire de la réanimation, préface de Jean-Claude Schmitt}

Ceyzérieu, Champ Vallon, coll. «Époques », 2018

Vincent Guigueno

\section{RÉFÉRENCE}

Anton Serdeczny, Du tabac pour le mort : une histoire de la réanimation, préface de Jean-

Claude Schmitt. Ceyzérieu, Champ Vallon, coll. «Époques », 2018, 400 p. 


\section{Du tabac}

\section{pour le mort}

\section{Une histoire de la réanimation}

Préface de Jean-Claude Schmitt

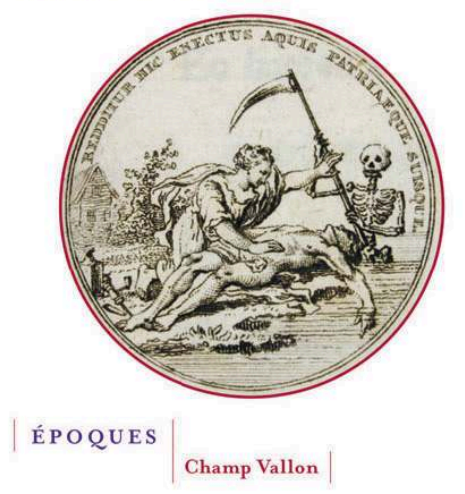

En 1794, l'apothicaire et médecin anglais William Hawes, connu pour ses travaux sur l'histoire de la réanimation des noyés à Londres, écrivait dans les Transactions of the Royal Human Society: "L'historien futur trouvera ici des raisons fondées de décrire le XVIII ${ }^{e}$ siècle comme l'âge du sauvetage de la vie ${ }^{1}$. » L'objet du livre d'Anton Serdeczny n'est pas l'étude historique ou sociologique des sociétés de secours aux noyés qui se développent au XVIII siècle, à Amsterdam puis à Londres, mais celle de la méthode préconisée pour les réanimer: la «fumigation de tabac par le fondement» ou « insufflation alvine " grâce à des «boîtes fumigatoires", dont la prolifération dans l'espace public n'est pas sans rappeler celle de nos actuels défibrillateurs².

2 Le parti pris de cette enquête est de prendre au sérieux cette pratique de réanimation déroutante, mais très répandue au XVIII ${ }^{\mathrm{e}}$ siècle, et avérée jusqu'à la fin du XIXe siècle. Celle-ci semble en contradiction avec l'esprit des Lumières, qui propose de fonder la médecine sur la raison. Or c'est bien la technique d'insufflation alvine qui est mise en avant dans l'Encyclopédie de Diderot et d'Alembert, où l'auteur du livre a découvert par hasard le sujet de sa thèse ${ }^{3}$. Pourquoi ce choix audacieux ? L'auteur explique que «le développement et l'enrichissement des approches de l'histoire des sciences laissent essentiellement en marge un phénomène de ce type » (p. 18). L'histoire de la médecine aurait donc mis de côté des récits sans rapport direct avec les connaissances contemporaines, en particulier sur les techniques de réanimation par insufflation buccale et massage cardiaque. Anton Serdeczny fait de cette pratique oubliée, effacée des manuels d'histoire, la pierre angulaire d'un travail fondé sur un vaste corpus de textes scientifiques et, de manière plus inattendue, sur des contes et légendes.

Le livre est divisé en quatre parties exposant une démonstration rigoureuse, où après avoir épuisé les éléments d'une explication internaliste de l'histoire des sciences, l'auteur se tourne vers des pistes externalistes particulièrement fécondes. La première 
partie est consacrée à la naissance d'un "paradigme réanimatoire" au XVIII siècle, dont l'auteur livre une généalogie précise à travers les écrits des savants des Lumières. Même si l'étude de la réanimation est observée à Rome au début du xvIII ${ }^{\mathrm{e}}$ siècle, Serdeczny soutient qu'elle se diffuse à partir de 1733 grâce aux publications virulentes de Louis Bourguet dans le Mercure suisse. Savant éclectique, théologien, archéologue, titulaire d'une chaire de philosophie et de mathématiques, Bourguet impute la noyade à une méconnaissance des gestes propres à réanimer les noyés, comme l'insufflation alvine ou l'acte d'uriner dans la bouche. Les savants - dont René Antoine Ferchault de Réaumur (1683-1757) - et les médecins font écho au discours de Bourguet. Ainsi, dans sa traduction commentée de la Dissertation sur l'incertitude des signes de la mort... (1742) de Jacob Benignus Winslow, Jacques-Jean Bruhier écrit : « Pour donner secours à ceux que l'on croit noïés [...] tout ce qu'il y a de mieux, peut-être, c'est de souffler dans les intestins la fumée du tabac d'une pipe ${ }^{4}$.» Dans l'abondante littérature du siècle des Lumières consacrée à la crainte d'enterrer les vivants - la taphophobie -, les méthodes de réanimation préconisées par Bourguet intègrent donc un ensemble de pratiques scientifiquement validées.

Dans une deuxième partie, Serdeczny cherche les origines de cette réanimation moderne défendue par les savants à partir de la seconde moitié du xvIII ${ }^{\mathrm{e}}$ siècle. Faut-il y voir la traduction médicale d'une mécanique du corps, telle qu'elle se développe dans la philosophie et les sciences de l'époque, le noyé étant alors une horloge arrêtée qu'il s'agirait de faire redémarrer? Le tableau (p.85) qui démontre une codification paradigmatique du discours suite aux publications de Bourguet de 1733 comporte en effet plusieurs "anomalies", comme les qualifie Serdeczny, c'est-à-dire des textes antérieurs à cette date charnière. Ces textes précurseurs le conduisent sur la piste de pasteurs protestants du XVII ${ }^{e}$ siècle, dont un certain Albinus, auteur en 1620 d'un Petit Traité sur les moyens de sauver un noyé, le premier ouvrage vernaculaire abordant le sujet. Cet ancrage confessionnel de la réanimation médicale au XVII siècle annonce son développement scientifique, puis sociétal, dans des régions de culture protestante: l'Angleterre, les Pays-Bas. La pratique réanimatoire n'est donc pas la marque d'une déchristianisation, comme l'écrit l'historien Claudio Milanesi, mais un acte de piété, une manifestation de la volonté de Dieu dont le sauveteur serait l'instrument. Cette deuxième partie s'achève par une réflexion plus générale inscrivant la réanimation des noyés, mais aussi celle des pendus et des asphyxiés, dans la construction d'une nouvelle relation à la mort qui " change de ton " au cours du XviII e siècle, selon une expression de Serdeczny inspirée par les travaux de Michel Vovelle ${ }^{5}$. Elle n'est plus un châtiment mais un phénomène naturel dont la perception et la "gestion " évoluent avec, par exemple, le déplacement des cimetières hors des villes.

5 Cette immersion - si l'on ose la métaphore - dans les discours savants consacrés à la réanimation ne dit pas grand-chose des pratiques de secours elles-mêmes, dont l'auteur offre un inventaire souvent savoureux dans la troisième partie du livre, intitulée « À la poursuite des gestes » : réchauffer le noyé avec des cendres ou du fumier, traitement qui fut réservé au connétable Du Guesclin après une chute dans les fosses de Melun, en 1359 ; l'envelopper dans la peau d'un mouton fraîchement écorché ; lui faire boire de l'urine chaude ; le rouler dans un tonneau ; lui raser la tête... Anton Serdeczny qualifie de «flottantes" ces pratiques qui ne relèvent pas de la médecine savante mais d'un empirisme insaisissable, même quandil s'agit de l'insufflation alvine, dont les méthodes et les instruments sont longuement décrits dans le texte. Le remplacement de l'air par 
du tabac trouve-t-il son origine chez les Amérindiens Micmac? Le récit du chirurgien normand Diéreville qui l'affirme semble plus que douteux pour Serdeczny.

L'auteur donne la clef qui lui permet d'interpréter les recommandations des savants des Lumières dans la quatrième partie du livre intitulée «Des contes au pays des Lumières ». Il ouvre "la porte de l'externalisme » avec la lecture d'un chapitre des Voyages de Gulliver de Jonathan Swift, où la pratique de l'insufflation alvine par un chirurgien est moquée de manière burlesque (p.251). Inspiré par ce récit, Anton Serdeczny formule l'hypothèse suivante : «L'insufflation savante [prend le sens d'une] réélaboration dans la médecine savante d'un geste symbolisant la résurrection dans le répertoire du carnaval», la danse des "soufflaculs" (p.258). Pour légitimer scientifiquement la pratique de la réanimation médicale, les savants ont puisé dans une culture populaire - contes, romans rabelaisiens, rituels carnavalesques - qui mime la résurrection. Dans cette perspective anthropologique, la chronologie se desserre, les sources se diversifient, allant des livres d'heures du xiII ${ }^{\mathrm{e}}$ siècle à des observations ethnographiques $\mathrm{du} \mathrm{xx}^{\mathrm{e}}$ siècle, attestant d'une très large diffusion de l'insufflation alvine, symbole du retour de l'âme des enfers. Au passage, le détour par le carnaval permet de comprendre la fausse attribution aux Micmac de l'insufflation de tabac, ceux-ci étant assimilés aux « hommes sauvages » carnavalesques dont les Amérindiens deviennent l'incarnation (p. 296-297). L'hypothèse d'un emprunt par les savants de motifs et de symboles venus des contes explique également la mise à contribution des oiseaux dans le discours sur la réanimation des noyés. La Dissertation sur l'incertitude des signes de la mort de Bruhier rapproche la réanimation des noyés par insufflation et les pratiques supposées d'hibernation subaquatique des hirondelles et des cigognes. Cette capacité attribuée aux oiseaux de passer du monde des vivants à celui des morts explique leur présence récurrente dans l'imaginaire de la résurrection des âmes.

7 En mobilisant une histoire des sciences désormais classique, c'est-à-dire attentive aux usages oubliés et ouverte à l'anthropologie, Anton Serdeczny explique la diffusion de pratiques de réanimation qui peuvent sembler comiques ou aberrantes. Il conclut qu'une pratique soutenue par des savants rationnels est inspirée par un système symbolique présent dans des contes et des rites de carnaval. Trouver des mythes au cœur du discours scientifique des Lumières revêt, aux yeux du spécialiste de l'anthropologie historique médiévale Jean-Claude Schmitt, une grande importance. Dans sa préface, ce dernier affirme que l'étude d'Anton Serdeczny «permet d'éclairer une étape cruciale de l'histoire des sciences [...] en plein basculement entre - pour adopter ici la terminologie de l'anthropologue Philippe Descola - la très ancienne tradition européenne de l'ontologie analogique et la nouvelle ontologie naturaliste qui [...] triomphera avec le matérialisme positiviste et technologique du $\mathrm{xIx}^{\mathrm{e}}$ et $\mathrm{xx}^{\mathrm{e}}$ siècles » (p.11). Cette étude éclaire en effet un "angle mort des Lumières ». L'écriture, d'une grande rigueur sans être dénuée d'humour, est une autre réussite de ce livre « funambule », entre histoire et anthropologie. 


\section{NOTES}

1. Voir William Hawes, The Transactions of the Royal Human Society, I Nichols, s.d.: 103, cite et traduit par Anton Serdeczny : 28.

2. Anton Serdeczny, "Souffler du tabac dans le derrière des noyés, les recouvrir de fumier, les histoires insolites de la réanimation ", Huffpost [en ligne], disponible sur: https:// www.huffingtonpost.fr/anton-serdeczny/souffler-du-tabac-dans-le-derriere-des-noyes-les-

recouvrir-de-fumier-les-histoires-insolites-de-la-reanimation_a_23551074/, consulté le 25 juillet 2019.

3. Voir Anton Serdeczny, «D'entre les morts : une anthropologie historique de la réanimation, $\mathrm{XVII}^{\mathrm{e}}$-XVIII ${ }^{\mathrm{e}}$ siècle ", thèse de doctorat en histoire moderne, Paris, EPHE, sous la direction d'olivier Christin, 2014. L'auteur en raconte la genèse dans un entretien donné à Nicolas Martin le $1^{\text {er }}$ novembre 2018 dans "La méthode scientifique », France Culture, disponible sur: https:// www.franceculture.fr/emissions/la-methode-scientifique/histoire-de-la-reanimation-quand-lamedecine-donne-un-second-souffle (consulté le 18 juin 2019).

4. Voir Jacob Benignus Winslow, Dissertation sur l'Incertitude des signes de la mort et l'abus des enterremens et embaumemens précipités, traduite et commentée par Jacques-Jean Bruhier. Paris, C.F. Simon, fils, $1742: 41$.

5. Voir Michel Vovelle, La Mort et l'Occident : de 1300 à nos jours. Paris, Gallimard, 2000.

\section{AUTEURS}

\section{VINCENT GUIGUENO}

Vincent.GUIGUENO[at]quaibranly.fr 